\title{
Recurrent and life-threatening strokes after pacemaker implantation in a patient affected by concealed superior sinus venosus atrial septal defect
}

\author{
Giusy Sirico ${ }^{1}$, Andrea Montisci ${ }^{2}$, Domenico Sirico ${ }^{3}$, Alfonso Ielasi ${ }^{4}$, \\ Michele Criscuolo ${ }^{5}$, Mauro Preziosa ${ }^{5}$, Maurizio Tespili ${ }^{4}$, Massimo Mantica $^{1}$ \\ ${ }^{1}$ Department of Cardiac Electrophysiology and Pacing, Istituto Clinico Sant'Ambrogio, Milan, Italy \\ ${ }^{2}$ Department of Anesthesia and Intensive Care, Cardiothoracic Center, \\ Istituto Clinico Sant'Ambrogio, Milan, Italy \\ ${ }^{3}$ Paediatric Cardiology Services, Royal Brompton Hospital and \\ Harefield NHS Foundation Trust, London, United Kingdom \\ ${ }^{4}$ Department of Clinical and Interventional Cardiology, Istituto Clinico Sant'Ambrogio, Milan, Italy \\ ${ }^{5}$ Department of Radiology, Istituto Clinico Sant'Ambrogio, Milan, Italy
}

\begin{abstract}
A 68-year-old woman was referred to the documented hospital after large and consecutive strokes following pacemaker implantation. Upon arrival she was aphasic, hemiplegic and lethargic. Electrocardiogram showed constant QRS stimulated in right bundle branch block pattern. Transthoracic echocardiography showed leads positioned in left heart with right chambers dilation and estimated pulmonary artery systolic pressure of $35 \mathrm{mmHg}$. Angiography with a pigtail catheter in the innominate vein showed overriding superior vena cava between right and left atria, with leads ending up inside the left heart (Suppl. Video 1, Fig. 1A, left). Lead removal was then performed to prevent further cardio-embolic strokes and new right chamber endocardial leads were implanted. Critical clinical conditions discouraged an epicardial pacing system implantation. Pacemaker depend-
\end{abstract}

ency compelled positioning the first new active fixation leads in right atrial appendage and right ventricular septum before left side lead removal. At left anterior oblique fluoroscopy four leads were temporarily present in four chambers of the heart (Fig. 1A, right) showing left sided leads posteriorly located. Previous leads were then extracted from left heart without complication. Final electrocardiogram showed stimulated QRS in a left bundle branch block pattern. Contrast chest computed tomography scan at sagittal plane (Fig. 1B, left) and three-dimensional reconstruction (Fig. 1B, right) showed concealed superior sinus venosus atrial septal defect with partial anomalous pulmonary venous return. Images herein highlight an occasional diagnosis of sinus venosus atrial septal defect following an inadvertent lead malposition associated to cerebral embolism.

Conflict of interest: None declared

Address for correspondence: Giusy Sirico, MD, PhD, Istituto Clinico Sant'Ambrogio, Via Giuseppe Faravelli 16, 20149 Milan, Italy, tel: +39-02-33127712, fax: +39-02-33127711, e-mail: giusy.sirico@gmail.com 


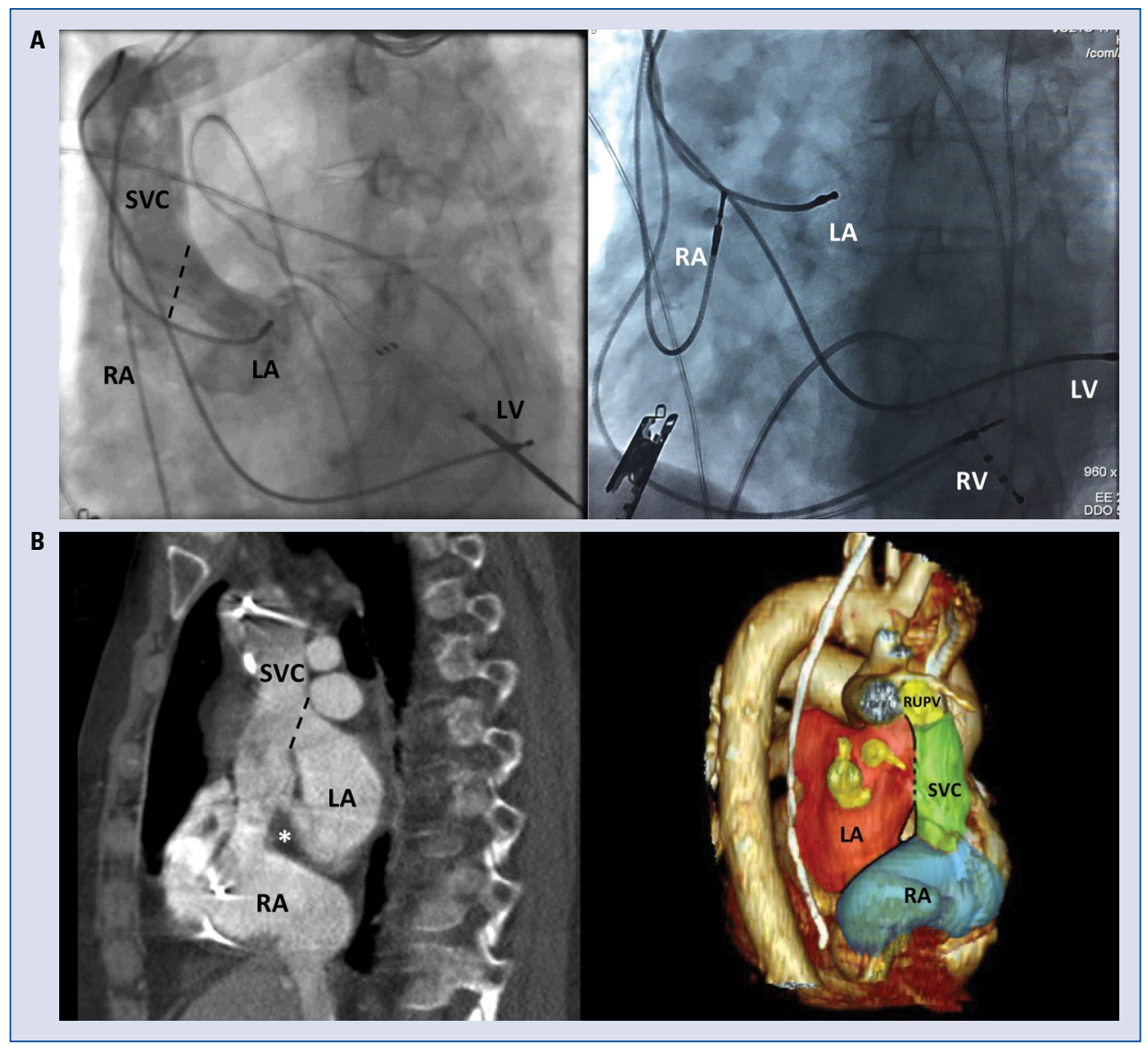

Figure 1. A. Superior vena cava (SVC) selective angiography (left) showing shunt between right and left atria through the sinus venosus atrial septal defect (SVASD) and left anterior oblique fluoroscopy (right) showing four leads temporarily present in four chambers of the heart; B. Chest computed tomography scan: sagittal plane (left) showing SVASD (dashed line) at the upper most part of inter atrial septum (asterisk) with SVC overriding the defect and threedimensional reconstruction (right) showing the position of the superior SVASD (dotted black line) and abnormal drainage of the right upper pulmonary vein (RUPV) to the SVC. Left atrium (LA) in red, right atrium (RA) in blue, SVC in green and RUPVs in yellow; LV — left ventricle; RV — right ventricle. 